\title{
Pre-Scaling Optimization for Space Shift Keying Based on Semidefinite Relaxation
}

\author{
Adrian Garcia-Rodriguez, Student Member, IEEE, Christos Masouros, Senior Member, IEEE, and \\ Lajos Hanzo, Fellow, IEEE
}

\begin{abstract}
The performance of space shift keying (SSK) is known to be dominated by the minimum Euclidean distance (MED) in the received SSK constellation. In this paper, we propose a method of enhancing the MED in the received SSK constellation and improving both the attainable performance and the power efficiency by means of symbol scaling at the transmitter. To this aim, we formulate a pair of optimization problems, one for maximizing the MED subject to a specific transmit power constraint and one for minimizing the transmit power subject to a MED threshold. As these problems are NP-hard, we re-formulate their optimization using semidefinite relaxations, which results in convex problem formulations that can be efficiently solved using standard approaches. Moreover, we design pre-scaling techniques for imperfect channel state information at the transmitter, where the existing approaches are inapplicable. Our results show that the proposed schemes substantially improve the power efficiency of SSK systems with respect to state-of-the-art techniques by offering an improved performance for specific transmit power requirements or, equivalently, a transmit power reduction for a given MED threshold.
\end{abstract}

Index Terms-Space shift keying, constellation shaping, multiple-input multiple-output, pre-scaling, imperfect CSI.

\section{INTRODUCTION}

$\mathbf{S}$ PACE SHIFT KEYING (SSK) along with its dual-domain adaptation, namely spatial modulation (SM) have been shown to offer a low complexity alternative to spatial multiplexing, where only a subset (down to one) of radio frequency (RF) chains are required for transmission [1]-[3]. Early work has focused on the design of receiver algorithms for minimizing the bit error rates (BERs) of SM at a low complexity [1], [2],

Manuscript received February 28, 2015; revised July 7, 2015; accepted August 16, 2015. Date of publication August 20, 2015; date of current version November 13, 2015. The work of C. Masouros was supported by the Royal Academy of Engineering, U.K., and the Engineering and Physical Sciences Research Council (EPSRC) project EP/M014150/1. The work of L. Hanzo was supported under the auspices of the India-U.K. Advanced Technology Centre (IU-ATC), of the EU's Concerto project as well as that of the European Research Council's Advanced Fellow Grant and that of the Royal Society's Wolfson Research Merit Award. The associate editor coordinating the review of this paper and approving it for publication was S. Gezici.

A. Garcia-Rodriguez and C. Masouros are with the Department of Electronic and Electrical Engineering, University College London, WC1E 7JE London, U.K. (e-mail: adrian.rodriguez.12@ucl.ac.uk; chris.masouros@ieee.org).

L. Hanzo is with the School of Electronics and Computer Science, University of Southampton, SO17 1BJ Southampton, U.K. (e-mail: lh@ecs.soton.ac.uk).

Color versions of one or more of the figures in this paper are available online at http://ieeexplore.ieee.org.

Digital Object Identifier 10.1109/TCOMM.2015.2470656
[4]-[8]. Matched filtering is shown to be a low-complexity technique for detecting the antenna index used for SM [1]. A maximum likelihood (ML) detector is introduced in [2] for reducing the complexity of classic spatial multiplexing ML detectors. Reduced-space sphere detection has also been conceived for SM in [5] for further complexity reduction.

In addition to receive-side processing, recent contributions have also proposed constellation shaping for SSK and SM [9]-[14]. Specifically, in [9] the transmit diversity of coded SM is analyzed for different spatial constellations, which represent the legitimate sets of activated transmit antennas (TAs). Furthermore, [10] discusses symbol constellation optimization for minimizing the BER. However, spatial- and symbol-constellation shaping are discussed separately in [9], [10]. A number of constellation shaping schemes [11]-[14] have also been proposed for SSK, where the information is only carried in the spatial domain, namely by the activated antenna index (AI). Other comparable signal processing strategies include the design of both robust and non-robust precoders for pre-processing aided $\mathrm{SM}$, in which all the antennas are used at the transmitter, and the receiver only detects the signals gleaned from a single antenna [15], [16]. Additionally, the energy-efficient scheme developed in [17] improves upon the conventional Hamming code-aided SSK [18] by determining the optimal a priori symbol probabilities and by providing a criterion for bit mapping based on the Huffman coding algorithm. This technique allows for a variable number of RF chains as a way of enhancing the Hamming distance of the transmit symbols in order to improve performance, but it does not modify the amplitude nor does it alter the phase of the transmitted signals [17]. Instead, conceiving beneficial pre-scaling strategies for traditional SSK transmission constitute the focus of this work, where only a single RF-chain is required at the transmitter.

Closely related literature has focused on shaping the received SSK and SM constellations by means of symbol pre-scaling at the transmitter, aiming for maximizing the minimum Euclidean distance (MED) in the received SSK and SM constellations [19]-[21]. The constellation shaping approach of [19], [20] aims for fitting the receive SM constellation to one of the existing optimal constellation formats in terms of its minimum distance, say to quadrature amplitude modulation (QAM). Due to the strict constellation fitting requirement imposed on both the amplitude and phase, this pre-scaling relies on the inversion of the channel coefficients. In the case of ill-conditioned channels, this substantially increases the power required by the transmit constellation. Therefore, scaling factors must be 
employed for normalizing the transmit power, which however reduces the received signal-to-noise ratio (SNR). This problem has been alleviated in [21], where a constellation shaping scheme based on phase-only scaling is proposed. Still, the constellation shaping used in the above schemes is limited in the sense that it only applies to multiple-input single-output (MISO) systems where a single symbol is received for each transmission and thus both the characterization and shaping of the receive SM constellation is simple.

The designs developed in [22], [23] propose the uneven allocation of the transmission power in SSK and GSSK systems for the sake of minimizing the error rates. However, the attainable performance improvements of [22], [23] are limited due to the restriction of solely considering the amplitude variations in the transmit signals. The design of a pre-scaling codebook dependent on the long-term CSI is proposed in [24] for improving the performance attained in correlated fading channels. Constellation randomization (CR) proposed in [25] relies on a transmit pre-scaling (TPS) scheme where a number $D$ of randomly generated complex TPS factors are calculated off-line, which are known to both the transmitter and receiver. The transmitter then selects that particular set of TPS factors, which yielded the specific receive constellation having the maximum achievable MED. The scheme was proven to considerably improve the transmit diversity of SM, while it is readily applicable to SSK transmission as well. Furthermore, the optimization problems considered in this paper were independently studied in [26], published after the submission of this work, where a pair of iterative algorithms was introduced to find the pre-scaling factors. However, the iterative algorithms proposed in [26] require obtaining the solution of multiple convex optimization problems before reaching convergence and are not designed to cope with imperfect CSI.

To further improve upon the above-mentioned approaches, in this paper we optimize the TPS vectors using convex optimization strategies. We formulate the relevant problems of a) MED maximization under specific transmit power constraints and b) transmit power minimization under a MED threshold. Since these optimization problems can be shown to be NP-hard, we re-formulate them by using semidefinite relaxation (SDR), which yields convex objective functions as well as optimization constraints, and therefore optimization problems that are readily solvable using standard schemes [27]. This enhances the approach introduced in [25], where the set of candidate pre-scaling factors are generated without considering the channel characteristics, while it reduces the computational complexity of [26], where a convex optimization problem must be solved per iteration. Moreover, since none of the abovementioned TPS designs consider the impact of having imperfect CSI at the transmitter, we further extend these designs to improve their robustness to CSI acquisition errors. These robust designs allow our TPS strategies to guarantee that the system performance requirements are satisfied in the presence of imperfect CSI at the transmitter. Our results show that the proposed optimization problems offer further improved power efficiency and robustness to imperfect CSI with respect to the SSK and SSK using the constellation randomization schemes developed in [20], [25].
For clarity, we summarize the contributions of this paper as follows:

1) We formulate the optimal TPS problems of i) maximizing the performance of SSK transmission under specific transmit power constraints and ii) reducing the transmission power for a given MED threshold. Subsequently, we propose semidefinite relaxations of the above optimization problems to facilitate the application of efficient convex solvers and improve the power efficiency of SSK transmission.

2) We render our TPS techniques resilient to imperfect CSI at the transmitter by designing optimization problems that offer improved robustness to CSI estimation errors. This constitutes an enhancement over existing pre-scaling strategies, where the performance requirements specified at the receivers are not guaranteed under the presence of imperfect CSI.

In the following, vectors (matrices) are denoted by boldface lower (upper) case letters. The superscripts $(\cdot)^{T}$ and $(\cdot)^{H}$ denote transpose and conjugate transpose respectively. The notation $\operatorname{Tr}(\cdot)$ designates the trace of a matrix and $\operatorname{diag}(\mathbf{x})$ represents the diagonal matrix with its diagonal elements taken from vector $\mathbf{x}$. $\mathbb{E}[\cdot]$ denotes the expectation while $\|\cdot\|$ refers to the Euclidean norm. Moreover, $|\cdot|$ denotes the cardinality of a set and $\operatorname{rank}(\cdot)$ is the rank of a matrix.

\section{Transmit Pre-Scaling for Space Shift Keying}

Consider a multiple-input multiple-output (MIMO) link, where the transmitter and receiver are equipped with $N_{t}$ and $N_{r}$ antennas respectively as shown in Fig. 1 . We focus our attention on the single RF chain SSK approach, where the transmit vector is in the all-but-one zero form $\mathbf{s}^{k}=[0, \ldots, 1, \ldots, 0]^{T}$, where 1 is the $k$-th element of $\mathbf{s}^{k}$ and $k$ represents the index of the activated TA (the index of the non-zero element in $\mathbf{s}^{k}$ ) conveying $\log _{2}\left(N_{t}\right)$ bits in the spatial domain. Clearly, since $\mathbf{s}^{k}$ is an all-zero vector apart from a single element, there is no inter-antenna interference.

For the per-antenna TPS approach represented in Fig. 1, which is the focus of this paper, the signal fed to each TA is scaled by a complex-valued coefficient $a_{k}, k \in 1, \ldots, N_{t}$, which we aim to design in this work. These pre-scaling coefficients must be compliant with the average power constraint of $\mathbb{E}\left[a_{k} a_{k}^{H}\right] \leq P_{t}$. In this work we consider a flat independent fading channel model varying from one realization to the other. Defining the MIMO channel vector as $\mathbf{H}$ with elements $h_{m, n}$ representing the complex channel coefficient between the $n$ th TA to the $m$-th receive antenna (RA), the received symbol vector can be written as

$$
\mathbf{y}=\mathbf{H A} \mathbf{s}^{k}+\mathbf{w}
$$

where $\mathbf{w} \sim \mathcal{C N}\left(\mathbf{0}, \sigma^{2} \mathbf{I}\right)$ is the additive white Gaussian noise (AWGN) component at the receiver, with $\mathcal{C N}\left(\mu, \sigma^{2}\right)$ denoting the circularly symmetric complex Gaussian distribution with mean $\mu$ and variance $\sigma^{2}$. Furthermore, $\mathbf{A}=\operatorname{diag}(\mathbf{a})$ is the TPS matrix with $\mathbf{a}=\left[a_{1}, a_{2}, \ldots, a_{N_{t}}\right]$. The diagonal structure of 


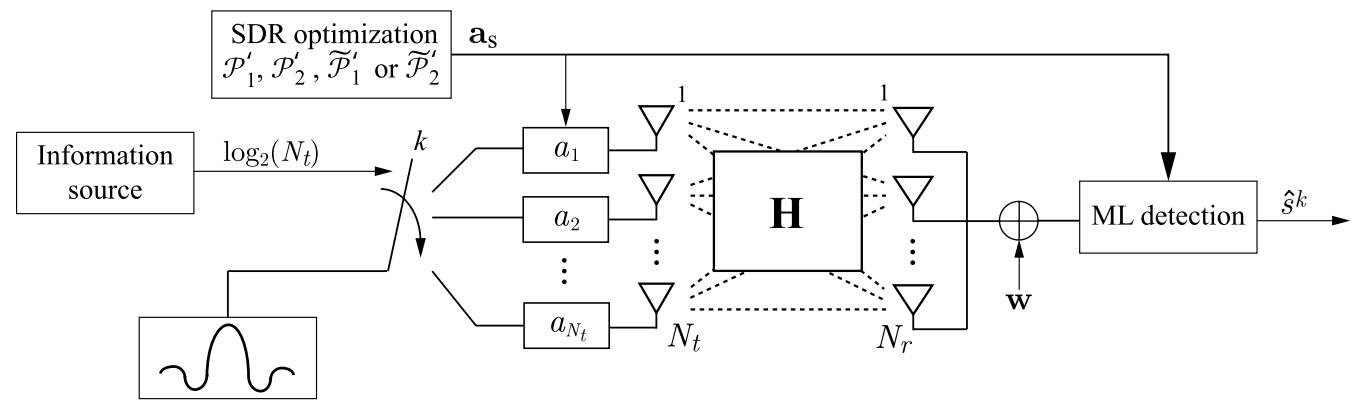

Fig. 1. Block diagram of SSK transceiver with pre-scaling and ML detection.

A guarantees having a transmit vector $\mathbf{t}=\mathbf{A} \mathbf{s}^{k}$ with a single non-zero element, so that the single-RF-chain benefit of SSK is preserved. We note that the pre-scaling coefficients are designed depending on the channel coefficients and not on the input bits, which is in contrast to conventional SM transmission [2].

At the receiver, the joint maximum likelihood (ML) detection of the TA index is achieved by the minimization of

$$
\begin{aligned}
\hat{k} & =\arg \min _{k}\left\|\mathbf{y}-\dot{\mathbf{y}}_{k}\right\|^{2} \\
& =\arg \min _{k}\left\|\mathbf{y}-\mathbf{H} \tilde{\mathbf{A}} \mathbf{s}^{k}\right\|^{2},
\end{aligned}
$$

where $\dot{\mathbf{y}}_{k}$ is the $k$-th constellation point in the received SSK constellation and $\widetilde{\mathbf{A}}$ refers to the TPS matrix estimated at the receiver. Note that, since the channel coefficients are estimated at the receiver for detection [1], [2], [4], [5], the pre-scaling designs of the following sections can be used both in the transmitter and in the receiver for deriving the same TPS matrix independently. Therefore no feed forwarding of the TPS vector a from the transmitter to the receiver is required. Indeed, when the channel coefficients are perfectly known both at the transmitter and receiver, they select the same TPS vector a independently, as per the optimization problems shown in the following. In other words, the transmitter must be aware of the TPS factors before transmission either via forwarding from the receiver or by their direct computation based on the available CSI. By exploiting the specific structure of the transmit vector, (2) can be further simplified to

$$
\hat{k}=\arg \min _{k}\left\|\mathbf{y}-\mathbf{h}_{k} a_{k}\right\|^{2},
$$

where $\mathbf{h}_{k}$ denotes the $k$-th column of the matrix $\mathbf{H}$. It is widely recognized that the performance of the detection as formulated above is dominated by the MED between the adjacent constellation points $\dot{\mathbf{y}}_{i}, \dot{\mathbf{y}}_{j}$ in the received SSK constellation [28], [29], which is formulated as:

$$
\begin{aligned}
d_{\text {min }} & =\min _{i, j}\left\|\dot{\mathbf{y}}_{i}-\dot{\mathbf{y}}_{j}\right\|^{2}, i \neq j \\
& =\min _{m, k}\left\|\mathbf{h}_{k} a_{k}-\mathbf{h}_{m} a_{m}\right\|^{2}, m \neq k .
\end{aligned}
$$

In the following we propose a TPS strategy for maximizing the above MED for a given transmit power budget considering perfect CSI availability. We then explore the related problem where the above expression is used as a constraint for minimiz- ing the transmit power [30], and further modify both designs for enhancing their robustness against imperfect CSI.

\section{A. Pre-Scaling Problem Formulation}

1) MED Maximization: Constellation shaping TPS schemes conceived for SSK aim for maximizing the MED in order to improve the likelihood of correct detection. Note that this objective differs from that of precoding in conventional MIMO transmission, where the availability of multiple active antennas is exploited to convey several data symbols across a number of active antennas [31]. Specifically, the optimal TPS vector $\mathbf{a}_{\text {opt }}$ can be found by solving the optimization problem

$$
\begin{aligned}
\mathcal{P}_{0}: & \underset{\mathbf{a}}{\operatorname{maximize}} \min _{\substack{m, k \\
m \neq k}}\left(\left\|\mathbf{h}_{k} a_{k}-\mathbf{h}_{m} a_{m}\right\|^{2}\right) \\
& \text { subject to } \operatorname{Tr}\left(\mathbf{a a}^{H}\right) \leq\left(P_{t} N_{t}\right) .
\end{aligned}
$$

Here, $k, m \in 1, \ldots N_{t}$, represent the index of the active antenna at the transmitter, $\mathbf{a} \in \mathbb{C}^{N_{t} \times 1}$ is the variable to optimize, and $P_{t}$ denotes the transmit power budget. Note that in SSK only a single antenna is active at any symbol-instant. Therefore, the constraint in the second line of (5) guarantees an average transmission power $P_{t}$. The above optimization problem is equivalent to maximizing an auxiliary variable $d$ representing the MED according to

$$
\begin{gathered}
\mathcal{P}_{1}: \underset{\mathbf{a}}{\operatorname{maximize}} d \\
\text { subject to }\left\|\mathbf{h}_{k} a_{k}-\mathbf{h}_{m} a_{m}\right\|^{2} \geq d, \forall m \neq k \\
\operatorname{Tr}\left(\mathbf{a a}^{H}\right) \leq\left(P_{t} N_{t}\right) .
\end{gathered}
$$

2) Power Minimization: A problem related to the above one involves the minimization of the transmit power subject to a predefined MED threshold $d$. This is found according to the optimization of:

$$
\begin{gathered}
\mathcal{P}_{2}: \underset{\mathbf{a}}{\operatorname{minimize}}\|\mathbf{a}\|^{2} \\
\text { subject to }\left\|\mathbf{h}_{k} a_{k}-\mathbf{h}_{m} a_{m}\right\|^{2} \geq d, \forall m \neq k .
\end{gathered}
$$

It can be seen that in the above formulations the quadratic constraints that represent the MED are not convex with respect to $\mathbf{a}$ [27]. This therefore makes finding the optimal solution of the problems $\mathcal{P}_{1}$ and $\mathcal{P}_{2}$ computationally challenging. 


\section{Pre-Scaling Using Semidefinite Relaxation}

The above optimization problems constitute nonconvex quadratically constrained quadratic programs, which have been shown to be NP-hard in general [27], [32]-[34]. This motivates the conception of a more efficient approach. In the following we explore the semidefinite relaxation (SDR) of the above problems in order to facilitate the employment of efficient solvers [32]. Hence, we first determine the number of constraints in the second line of $\mathcal{P}_{1}$ and $\mathcal{P}_{2}$. In particular, the number of nonidentical constraints is given by

$$
N_{c}=\sum_{t=1}^{N_{t}}\left(N_{t}-t\right)=N_{t}\left(N_{t}-1\right) / 2,
$$

where we have used the fact that for any $x_{i}, x_{j}, i \neq j$, the operation $\left\|x_{i}-x_{j}\right\|$ is equivalent to $\left\|x_{j}-x_{i}\right\|$, which makes it unnecessary to analyze the symmetrical terms.

Next we focus our attention on the reformulation of the optimization constraints. For simplicity, let us commence with the case of $N_{r}=1$, so that we have $\mathbf{h}_{k}=h_{k}$. At this point we would like to point out that the MED constraints resemble the ones conventionally employed for establishing a minimum distance between the nodes in the sensor network location problem [27], [35]. In accordance with the above, the $j^{t h}$ constraint in $\mathcal{P}_{2}, c(j)$, can be reformulated as [35]

$$
c(j)=\operatorname{Tr}\left(\mathbf{C}_{j} \mathbf{a}^{H}\right)=\operatorname{Tr}\left(\left(\mathbf{e}(j)_{k_{j}}^{m_{j}}\right)^{H} \mathbf{e}(j)_{k_{j}}^{m_{j}} \mathbf{a}^{H}\right),
$$

where the constraints vectors $\mathbf{e}(j)_{k_{j}}^{m_{j}}$ have two non-zero entries in the indices $k_{j}, m_{j}$, which are given by

$$
\mathbf{e}(j)_{k_{j}}^{m_{j}}=\left[0, \ldots, h_{k_{j}}, \ldots,-h_{m_{j}}, \ldots, 0\right] .
$$

Here, $k_{j}, m_{j} \in 1, \ldots N_{t}$, represent the indices of the transmit antennas considered in the $j$-th constraint.

For $N_{r}>1$, the left-hand side of the optimization constraints can be decomposed as

$$
\left\|\mathbf{h}_{k} a_{k}-\mathbf{h}_{m} a_{m}\right\|^{2}=\sum_{t=1}^{N_{r}}\left\|h_{(k, t)} a_{k}-h_{(m, t)} a_{m}\right\|^{2},
$$

where $h_{(k, t)}$ denotes the $t$-th entry of $\mathbf{h}_{k}$. This allows us to reformulate the optimization constraints as a linear combination of the constraints derived in (9), yielding:

$$
\begin{aligned}
c(j) & =\sum_{t=1}^{N_{r}} \operatorname{Tr}\left(\mathbf{C}_{j, t} \mathbf{a}^{H}\right)=\operatorname{Tr}\left(\mathbf{a a}^{H} \sum_{t=1}^{N_{r}} \mathbf{C}_{j, t}\right) \\
& =\operatorname{Tr}\left(\mathbf{a a}^{H} \mathbf{C}_{j}^{\prime}\right) .
\end{aligned}
$$

Here, $\mathbf{C}_{j, t}, t \in 1, \ldots N_{r}$, represents the constraint matrix for the $t$-th receive antenna as shown in the right-hand side of (9)

$$
\mathbf{C}_{j, t}=\left(\mathbf{e}(j)_{\left(k_{j}, t\right)}^{\left(m_{j}, t\right)}\right)^{H} \mathbf{e}(j)_{\left(k_{j}, t\right)}^{\left(m_{j}, t\right)},
$$

with

$$
\mathbf{e}(j)_{\left(k_{j}, t\right)}^{\left(m_{j}, t\right)}=\left[0, \ldots, h_{\left(k_{j}, t\right)}, \ldots,-h_{\left(m_{j}, t\right)}, \ldots, 0\right],
$$

and we have defined the $j$-th constraint matrix as

$$
\mathbf{C}_{j}^{\prime} \triangleq \sum_{t=1}^{N_{r}} \mathbf{C}_{j, t} .
$$

Note that the constraint matrices $\mathbf{C}_{j}^{\prime}$ are sparse by definition, an aspect that will be considered in the robust design to be introduced in Section IV. Overall, the above representation allows us to re-formulate the optimization problems $\mathcal{P}_{1}$ and $\mathcal{P}_{2}$ as shown in the following.

\section{A. MED Maximization}

Based on the above and noting that $\|\mathbf{a}\|^{2}=\operatorname{Tr}\left(\mathbf{a a}^{H}\right), \mathcal{P}_{1}$ can be reformulated as

$$
\begin{aligned}
& \mathcal{P}_{1}: \underset{\mathbf{X}}{\operatorname{maximize}} d \\
& \text { subject to } \operatorname{Tr}\left(\mathbf{C}_{j}^{\prime} \mathbf{X}\right) \geq d, \quad j=1, \ldots N_{c}, \\
& \\
& \operatorname{Tr}(\mathbf{X}) \leq\left(P_{t} N_{t}\right) \\
& \mathbf{X} \succeq 0, \operatorname{rank}(\mathbf{X})=1,
\end{aligned}
$$

where $\mathbf{X} \triangleq \mathbf{a a}^{H}$ and $\mathbf{X} \succeq 0$ denotes that $\mathbf{X}$ is a positive semidefinite matrix. This is a problem equivalent to (6) that yields the same solutions, and it is also NP-hard. However, the nonconvex rank constraint $\operatorname{rank}(\mathbf{X})=1$ can be dropped to obtain a convex semidefinite approximation to $\mathcal{P}_{1}$ [34]. Accordingly, we have the following SDR formulation

$$
\begin{aligned}
\mathcal{P}_{1}^{\prime}: \underset{\mathbf{X}}{\operatorname{maximize}} d & \\
\text { subject to } & \operatorname{Tr}\left(\mathbf{C}_{j}^{\prime} \mathbf{X}\right) \geq d, \quad j=1, \ldots, N_{c}, \\
& \operatorname{Tr}(\mathbf{X}) \leq\left(P_{t} N_{t}\right) \\
& \mathbf{X} \succeq 0 .
\end{aligned}
$$

The above is a standard convex optimization problem that can be efficiently solved using standard approaches [32].

\section{B. Power Minimization}

For the case of power minimization, a similar approach can be employed to reformulate the original problem $\mathcal{P}_{2}$ to

$$
\begin{aligned}
\mathcal{P}_{2}: \underset{\mathbf{X}}{\operatorname{minimize}} & \operatorname{Tr}(\mathbf{X}) \\
\text { subject to } & \operatorname{Tr}\left(\mathbf{C}_{j}^{\prime} \mathbf{X}\right) \geq d, \quad j=1, \ldots, N_{c}, \\
& \mathbf{X} \succeq 0, \operatorname{rank}(\mathbf{X})=1 .
\end{aligned}
$$

By dropping the rank constraint, the resultant SDR formulation can be expressed as

$$
\begin{aligned}
\mathcal{P}_{2}^{\prime}: \underset{\mathbf{X}}{\operatorname{minimize}} & \operatorname{Tr}(\mathbf{X}) \\
\text { subject to } & \operatorname{Tr}\left(\mathbf{C}_{j}^{\prime} \mathbf{X}\right) \geq d, \quad j=1, \ldots, N_{c}, \\
& \mathbf{X} \succeq 0 .
\end{aligned}
$$



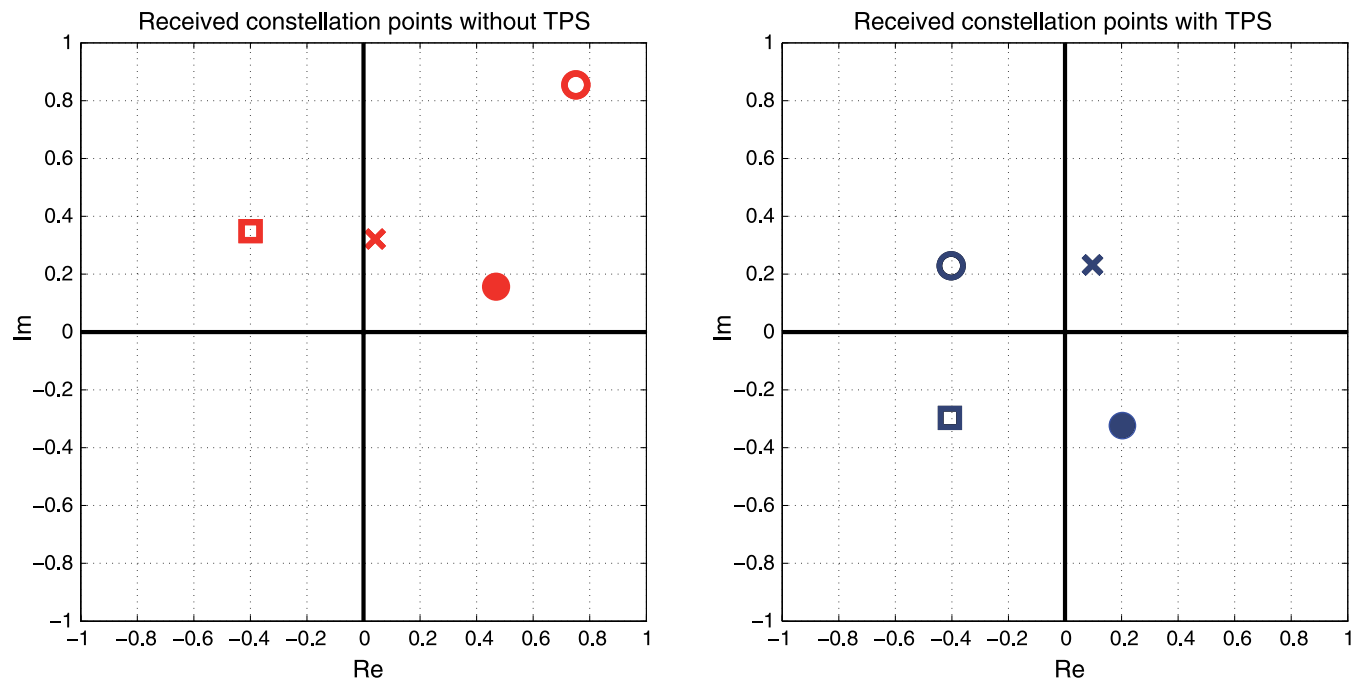

Fig. 2. Example of received constellation both with and without TPS for a $(4 \times 1)$ MISO system.

\section{Effect of the Optimization on the Received Constellation}

Fig. 2 shows the received constellation both with and without TPS for the illustrative example of a $(4 \times 1)$ MISO system. In this figure, the TPS scenario refers to the proposed prescaling using the optimization $\mathcal{P}_{2}^{\prime}$ and the different constellation symbols are represented with distinct geometrical figures. The convex optimization problems formulated throughout this paper have been implemented and solved with the aid of the standard toolbox conceived for disciplined convex programming CVX [36]. This figure shows that there is a substantial enhancement in both the MED and transmit power between the two constellations of Fig. 2. It can be seen that the random distribution of the receive constellation points for conventional SSK has been reshaped into a more regular constellation that roughly resembles a QAM constellation. Despite this improvement in the MED, the solution to $\mathcal{P}_{2}$ is not strictly optimal due to the removal of the rank constraint, which motivates the study of this effect in the following section.

\section{Analyzing the Impact of the Problem Relaxation}

It is widely recognized that the solution retrieved by the convex solver $\mathbf{X}_{s}$ with $\mathrm{SDR}$ is only optimal only when we have $\operatorname{rank}\left(\mathbf{X}_{s}\right)=1$ [27]. In this case the pre-scaling vectors, $\mathbf{a}_{s}$, can simply be obtained by using the principal component of the optimal solution $\mathbf{X}_{s}$ of problems $\mathcal{P}_{1}^{\prime}$ and $\mathcal{P}_{2}^{\prime}$, hence guaranteeing $\mathbf{X}_{s}=\mathbf{a}_{\text {opt }} \mathbf{a}_{\text {opt }}^{H}$, where $\mathbf{a}_{\text {opt }}$ refers to the optimal TPS vector. In the case where the solver does not return a rank-1 solution, randomization techniques can be employed for promoting the appearance of close-to-optimal low-rank solutions and for obtaining the pre-scaling vectors [32]. Here, we utilize the approach of [32]. Let the eigen-decomposition of the solution retrieved by the solver $\mathbf{X}_{s}$ be defined as $\mathbf{X}_{s}=$ $\mathbf{U} \boldsymbol{\Sigma} \mathbf{U}^{H}$, where $\mathbf{U}$ is the $\left(N_{t} \times N_{t}\right)$-element square matrix whose $i$-th column is the eigenvector $\mathbf{u}_{i}$ of $\mathbf{X}_{s}$ and $\boldsymbol{\Sigma}$ is the diagonal matrix with the eigenvalues of $\mathbf{X}_{s}$ on its diagonal. We construct the candidate pre-scaling vectors as $\mathbf{a}_{s}=c \mathbf{U} \Sigma^{1 / 2} \mathbf{v}$, where the elements $v_{i}$ of $\mathbf{v}$ are independent random variables $v_{i}=e^{j \theta_{i}}$, uniformly distributed on the unit circle of the complex plane with $\theta_{i}$ being independent and uniformly distributed in $(0,2 \pi]$ [32]. The scaling constant $c$ is selected for ensuring that the original problem constraints are satisfied [32]. Note that the employment of principal component selection or randomization techniques when $\operatorname{rank}\left(\mathbf{X}_{s}\right) \neq 1$ implies that $\mathbf{A}_{s} \triangleq \mathbf{a}_{s} \mathbf{a}_{s}^{H} \neq$ $\mathbf{a}_{\text {opt }} \mathbf{a}_{\text {opt }}^{H}$, which in turn penalizes the value of the objective function.

An ideal characterization of the effects of the above degradation should be based on comparing the values of the objective function obtained by the proposed relaxations $\mathcal{P}_{1}^{\prime}, \mathcal{P}_{2}^{\prime}$ after randomization with the ones of the original optimal problems $\mathcal{P}_{1}, \mathcal{P}_{2}$. However, since the original problems are NP-hard, determining the optimal solution is practically infeasible in most cases. Following [32], [37], [38], we exploit the fact that the SDR problems provide a readily computable bound of the true solutions. For instance, if $f_{1}^{\star}$ and $f_{1}^{\prime \star}$ are the values of the objective functions for the MED maximization problems $\mathcal{P}_{1}$ and $\mathcal{P}_{1}^{\prime}$ respectively, using the solutions returned by the solver, then $f_{1}^{\star} \leq f_{1}^{\prime \star}$ holds, i.e. the semidefinite relaxation offers an upper bound of the original optimization problem's true solution. Based on the above and following [32], [37], [38], we define a relevant figure of merit $\mathcal{F}$ as

$$
\mathcal{F}_{\{1,2\}}=\frac{f_{\{1,2\}}^{\prime \star}}{f_{\{1,2\}}^{s}} .
$$

Here, $f_{\{1,2\}}^{\prime \star}$ represents the value of the objective function in $\mathcal{P}_{\{1,2\}}^{\prime}$ when the solution returned by the solver $\mathbf{X}_{s}$ is used, whereas $f_{\{1,2\}}^{s}$ denotes the value of the same function after randomization or principal component selection, i.e. by using $\mathbf{A}_{s}=\mathbf{a}_{s} \mathbf{a}_{s}^{H}$. The same metric can also be applied to both conventional SSK and to CR-aided SSK (SSK-CR) [25] to determine the proximity to an optimal solution.

Both the mean and standard deviation of $\mathcal{F}_{1}$ found for the proposed scheme using optimization $\mathcal{P}_{1}^{\prime}$, termed as SSK-SDR, 
TABLE I

$\operatorname{Mean}(\mu)$ And Standard Deviation $(\sigma)$ of $\mathcal{F}_{1}$ With $P_{t}=1$

\begin{tabular}{|c||c|c||c|c||c|c|}
\hline \multicolumn{1}{|c||}{$N_{t} / N_{r}$} & \multicolumn{2}{c|}{ SSK-SDR } & \multicolumn{2}{c||}{ SSK-CR } & \multicolumn{2}{c|}{ SSK } \\
\hline \hline & $\mu$ & $\sigma$ & $\mu$ & $\sigma$ & $\mu$ & $\sigma$ \\
\hline $2 / 1$ & 1 & 0 & 1.27 & 0.39 & 6.35 & 21.1 \\
\hline $4 / 2$ & 1.4 & 0.37 & 1.96 & 0.55 & 4.66 & 9.8 \\
\hline $4 / 3$ & 1.31 & 0.32 & 1.8 & 0.43 & 3.03 & 2.61 \\
\hline
\end{tabular}

for conventional SSK, and for SSK-CR with $D=20$ candidate scaling vectors are shown in Table I for different number of transmit and receive antennas. The number $D$ of candidate factors refers to the size of the set of random TPS vectors considered by the SSK-CR scheme [25]. In this case, $f_{1}^{s}$ and $f_{1}^{\prime \star}$ represent the MED in the received SSK constellation and, for a fair comparison, the average transmission power is set to $P_{t}=1$ for all techniques. Note that in this case we have $\mathcal{F}_{1} \geq 1$, since the MED for the considered strategies obtained by using $\mathbf{A}_{s}=\mathbf{a}_{s} \mathbf{a}_{s}^{H}$ is always smaller than or equal to the bound computed with the solution retrieved by the solver $\mathbf{X}_{s}$. The results show that both the mean and standard deviation of the figure of merit $\mathcal{F}$ are considerably reduced when the proposed pre-scaling is used, i.e. the solutions achieved by the relaxation advocated are closer to the optimal solution of $\mathcal{P}_{1}$ than the ones obtained for conventional SSK and SSK-CR. Specifically, the results of Table I allow us to conclude that the MED loss imposed by the relaxation procedure is usually in the range of $2-3 \mathrm{~dB}$ for a $4 \times 2 \mathrm{MIMO}$ system. This is because the distance of the solution obtained after relaxation from the optimal solution $f_{1}^{\star}$ is always smaller than the distance from its upper bound $f_{1}^{\prime \star}$ [32]. In fact, for the case of $N_{t}=2$ and $N_{r}=1$, it can be seen that the proposed relaxation always finds the optimal solution of the original problem $\mathcal{P}_{1}$, since the relaxed problem $\mathcal{P}_{1}^{\prime}$ always obtains rank-1 solutions. This is a direct consequence of the Shapiro-Barvinok-Pataki result, which states that the solution obtained by relaxing the original problem satisfies $\operatorname{rank}(\mathbf{X}) \leq 1$ when $N_{c} \leq 2$, i.e., under these conditions the relaxation is tight and the optimal solution can be efficiently found [27], [39]-[41].

\section{Robust Design: Worst-CASe Robustness TO IMPERFECT CSI}

The acquisition of perfect CSI is generally infeasible due to the presence of noise or finite quantization [31], an aspect not considered in the pre-scaling designs developed in [19]-[21], [25] and Section III. As a consequence, the performance of TPS techniques may be dramatically affected, since the actual received constellation symbols will be different from the ones estimated at the transmitter. For this reason, in this section we conceive robust designs to guarantee the target performance of the system under imperfect CSI conditions. In particular, we concentrate on the design of schemes based on preserving the required performance for the worst case of a bounded CSI error. This illustrative criterion has been selected due to the practicality of considering a given CSI acquisition error, above which the system's performance is no longer guaranteed [42]. With this objective, we model the estimated channel between the transmit antennas and the $m$-th receive antenna with imperfect CSI as [31]

$$
\widetilde{\mathbf{h}}_{m}=\sqrt{1-\tau^{2}} \mathbf{h}_{m}+\tau \mathbf{q}_{m},
$$

where $\widetilde{\mathbf{h}}_{m} \in \mathbb{C}^{N_{t} \times 1}$ denotes the imperfect CSI estimate available at the transmitter and $\mathbf{q}_{m} \sim \mathcal{C N}(\mathbf{0}, \mathbf{I})$. Here, $\tau$ is a parameter that determines the quality of the instantaneous acquired CSI ranging from perfect CSI $(\tau=0)$ to the unavailability of the instantaneous CSI $(\tau=1)$.

Clearly, following (13) and (14), the non-availability of perfect CSI should impact the definition of the constraint matrices $\mathbf{C}_{j}^{\prime}$ in (15) in order to meet the performance target at the receiver. To account for this, in this paper we exploit the principles behind robust beamforming designs to ensure that the target performance of the system is preserved [42]. In particular, we express the $j$-th constraint matrix of the robust pre-scaling design as [43]

$$
\overline{\mathbf{C}}_{j}=\sum_{t=1}^{N_{r}}\left(\widetilde{\mathbf{C}}_{j, t}+\boldsymbol{\Delta}_{j, t}\right)=\widetilde{\mathbf{C}}_{j}^{\prime}+\boldsymbol{\Delta}_{j},
$$

where the CSI uncertainty per receive antenna is modeled via an error matrix $\boldsymbol{\Delta}_{j, t}$ with a bounded Frobenius norm $\left\|\boldsymbol{\Delta}_{j, t}\right\|_{F} \leq$ $\epsilon_{j, t}$. For ease of notation and without loss of generality, in the following we consider $\boldsymbol{\Delta}_{j} \triangleq \sum_{t=1}^{N_{r}} \boldsymbol{\Delta}_{j, t}$ and $\left\|\boldsymbol{\Delta}_{j}\right\|_{F} \leq \epsilon_{j}$. The constant that upper-bounds the Frobenius norm of the error $\epsilon_{j}$ establishes the target performance depending on the accuracy of the available CSI, i.e. the parameter $\tau$ in (21). Moreover, $\widetilde{\mathbf{C}}_{j, t}$ corresponds to the $j$-th constraint matrix for the $t$-th receive antenna and, similarly to (13), (14), it is given by

$$
\widetilde{\mathbf{C}}_{j, t}=\left(\widetilde{\mathbf{e}}(j)_{\left(k_{j}, t\right)}^{\left(m_{j}, t\right)}\right)^{H} \widetilde{\mathbf{e}}(j)_{\left(k_{j}, t\right)}^{\left(m_{j}, t\right)},
$$

where $\widetilde{\mathbf{e}}(j)_{\left(k_{j}, t\right)}^{\left(m_{j}, t\right)}$ can be expressed as

$$
\widetilde{\mathbf{e}}(j)_{\left(k_{j}, t\right)}^{\left(m_{j}, t\right)}=\left[0, \ldots, \widetilde{h}_{\left(k_{j}, t\right)}, \ldots,-\widetilde{h}_{\left(m_{j}, t\right)}, \ldots, 0\right] .
$$

At this point we note that the sparse structure of $\widetilde{\mathbf{C}}_{j}^{\prime}$ must be incorporated into the definition of the error constraint matrix $\boldsymbol{\Delta}_{j}$. Intuitively, this is because the CSI estimation errors can only affect the non-zero entries of the $j$-th constraint matrix $\widetilde{\mathbf{C}}_{j}^{\prime}$. To account for this we define

$$
\mathcal{C}_{j}=\left\{\mathbf{C} \in \mathbb{C}^{N_{t} \times N_{t}} \mid \mathbf{C}_{\{r, c\}}=0 \forall\left(r \neq k_{j}, m_{j} \wedge c \neq k_{j}, m_{j}\right)\right\}
$$

as the set of sparse matrices with non-zero entries determined by $k_{j}$ and $m_{j}$, which correspond to the indices of the transmit antennas involved in the $j$-th constraint, as detailed in Section III. In the above expression, $\mathbf{C}_{\{r, c\}}$ denotes the $(r, c)$-th entry of the matrix $\mathbf{C}$ and $\wedge$ represents the logical function "and". Clearly, we have $\widetilde{\mathbf{C}}_{j}^{\prime} \in \mathcal{C}_{j}$ and $\boldsymbol{\Delta}_{j} \in \mathcal{C}_{j}$. In the following we concentrate on obtaining robust convex formulations of the relevant problems considered in this paper. 


\section{A. MED Maximization}

Following a procedure similar to the one used for deriving $\mathcal{P}_{1}^{\prime}$, the NP-hard robust pre-scaling optimization problem that maximizes the MED at reception subject to a total power constraint can be expressed as

$$
\begin{aligned}
\widetilde{\mathcal{P}}_{1}: \underset{\mathbf{X}}{\operatorname{maximize}} & d \\
\text { subject to } & \min _{\left\|\boldsymbol{\Delta}_{j}\right\|_{F} \leq \epsilon_{j}}\left\{\operatorname{Tr}\left(\left(\widetilde{\mathbf{C}}_{j}^{\prime}+\boldsymbol{\Delta}_{j}\right) \mathbf{X}\right)\right\} \geq d, \\
& \widetilde{\mathbf{C}}_{j}^{\prime}+\boldsymbol{\Delta}_{j} \succeq 0, \boldsymbol{\Delta}_{j} \in \mathcal{C}_{j}, j=1, \ldots, N_{c} . \\
& \operatorname{Tr}(\mathbf{X}) \leq\left(P_{t} N_{t}\right), \\
& \mathbf{X} \succeq 0, \operatorname{rank}(\mathbf{X})=1 .
\end{aligned}
$$

Here, we note that the constraint matrices of the optimization problem (26), $\widetilde{\mathbf{C}}_{j}^{\prime}+\boldsymbol{\Delta}_{j}$, differ from those of the conventional problem defined in (16). Specifically, the constraints consider the worst-case scenario in which the Euclidean distance of the received symbols is minimized for the matrices $\boldsymbol{\Delta}_{j} \in \mathcal{C}_{j}$ satisfying $\left\|\boldsymbol{\Delta}_{j}\right\|_{F} \leq \epsilon_{j}$. Additionally, the fact that the matrices $\mathbf{C}_{j}^{\prime}+\boldsymbol{\Delta}_{j}$ are positive semidefinite, which follows from their definition in (22)-(24), has also been considered, since it generally leads to better solutions [42], [43]. Since we are interested in finding a robust optimization problem that can be efficiently solved by applying standard convex optimization techniques, the following theorem provides a SDR version of $\widetilde{\mathcal{P}}_{1}$.

Theorem 1: Let $\mathbf{X} \triangleq \mathbf{a a}^{H}$, where a represents the pre-scaling vectors to be optimized. Moreover, let $\widetilde{\mathbf{C}}_{j}^{\prime}, j \in 1, \ldots, N_{c}$, be as defined in (22)-(24), while $\epsilon_{j}, j \in 1, \ldots, N_{c}$, and $d$ be predetermined constants. Then, a SDR version of $\widetilde{\mathcal{P}}_{1}$ in (26) is given by

$$
\begin{aligned}
\widetilde{\mathcal{P}}_{1}^{\prime}: & \underset{\mathbf{X}, \mathbf{B}_{j}}{\operatorname{maximize}} d \\
& \text { s.t. }-\epsilon_{j}\left\|\mathbf{B}_{j}-\mathbf{X}\right\|_{F}-\operatorname{Tr}\left(\widetilde{\mathbf{C}}_{j}^{\prime}\left(\mathbf{B}_{j}-\mathbf{X}\right)\right) \geq d, \\
& \mathbf{B}_{j} \succeq 0, \mathbf{B}_{j} \in \mathcal{B}_{j}, j=1, \ldots, N_{c}, \\
& \mathbf{X} \succeq 0, \operatorname{Tr}(\mathbf{X}) \leq\left(P_{t} N_{t}\right),
\end{aligned}
$$

where the optimization variables $\mathbf{B}_{j}, j \in 1, \ldots, N_{c}$, are the Lagrangian multipliers of the inner optimization problem contained in $\widetilde{\mathcal{P}}_{1}$, while $\mathcal{B}_{j}$ is the set of matrices satisfying $\mathcal{B}_{j}=$ $\left\{\mathbf{B}_{j} \in \mathbb{C}^{N_{t} \times N_{t}} \mid \mathbf{B}_{j,\{r, c\}}=\mathbf{X}_{\{r, c\}}\right.$ if $\left.\widetilde{\mathbf{C}}_{j,\{r, c\}}^{\prime}=0\right\}$, as detailed in Appendix A. Finally, the non-convex constraint of $\operatorname{rank}(\mathbf{X})=1$ has been dropped.

Proof: The proof is given in Appendix A.

The robust optimization problem $\widetilde{\mathcal{P}}_{1}^{\prime}$ can be solved with the aid of standard convex techniques and it facilitates the design of pre-scaling vectors robust to imperfect CSI conditions, as shown in Section VI.

\section{B. Power Minimization}

A similar procedure to the one detailed in Appendix A can be followed to obtain a relaxed robust version of the optimiza- tion problem that minimizes the transmission power subject to satisfying the performance thresholds $\mathcal{P}_{2}$, which can be expressed as

$$
\begin{aligned}
& \widetilde{\mathcal{P}}_{2}^{\prime}: \underset{\mathbf{X}, \mathbf{B}_{j}}{\operatorname{minimize}} \operatorname{Tr}(\mathbf{X}) \\
& \text { s.t. }-\epsilon_{j}\left\|\mathbf{B}_{j}-\mathbf{X}\right\|_{F}-\operatorname{Tr}\left(\widetilde{\mathbf{C}}_{j}^{\prime}\left(\mathbf{B}_{j}-\mathbf{X}\right)\right) \geq d, \\
& \quad \mathbf{B}_{j} \succeq 0, \mathbf{B}_{j} \in \mathcal{B}_{j}, j=1, \ldots, N_{c}, \\
& \quad \mathbf{X} \succeq 0 .
\end{aligned}
$$

At this point we remark that the original application of SDR to SSK developed in Section III facilitates the design of TPS schemes exhibiting robustness against imperfect CSI. We also note that with respect to the existing robust designs found in the literature, such as the downlink beamforming schemes of [42], [44], the constraint matrices $\widetilde{C}_{j}^{\prime}$ of the proposed designs (27) and (28) depend on the actual channel realization and not on its second-order statistics [42]. This is because we use the instantaneous MED at the receiver as a performance metric instead of the conventional average SNR, an approach that is particularly suited to SSK transmission that substantially modifies the definition of the relevant constraint matrices [19]-[21], [42], [44].

\section{Attainable Transmit Diversity and PERFORMANCE TRENDS}

While the transmit diversity order of the single-RF SSK is known to be one [9], the proposed TPS introduces an amplitude-phase diversity in the transmission, which is an explicit benefit of the optimization of the TPS factors and that of the positions of the constellation symbols in the receive SSK constellation. Accordingly, it was shown in [21], [25] that the attainable transmit diversity order depends on the average MED improvement of the pre-scaling as

$$
G \triangleq \frac{\mathbb{E}\left[d_{o}\right]}{\mathbb{E}\left[\min _{m, k}\left\|\mathbf{H s}^{k}-\mathbf{H s}^{m}\right\|^{2}\right]},
$$

where $d_{o}$ is the MED obtained with the aid of the proposed optimization problems $\mathcal{P}_{1}^{\prime}$ and $\mathcal{P}_{2}^{\prime}$. Intuitively, the above expression exploits that the diversity order directly depends on the pairwise error probability (PEP) and that, simultaneously, the largest PEP is determined by the MED between the received symbols [21], [25], [28], [29]. The derivation of the explicit connection is still an open problem [21], [25]. It is clear that for the case of $\mathcal{P}_{1}^{\prime}$ this depends on the transmit power budget and for $\mathcal{P}_{2}^{\prime}$ it is determined by the MED threshold. Additionally, SSK systems associated with $N_{r}$ uncorrelated RAs have been shown to experience a transmit diversity order of one and receive diversity order of $N_{r}$. Accordingly, since the proposed scheme attains a transmit diversity order of $G$, the total diversity order becomes $\delta=N_{r} G$. The resultant probability of error $P_{e}$ follows the high-SNR trend formulated as

$$
P_{e}=\alpha \gamma^{-N_{r} G},
$$


where $\gamma$ is the average SNR defined as $\mathrm{SNR}=P_{t} / \sigma^{2}$, and $\alpha$ is an arbitrary coefficient. In the following we show that the above provides a close approximation to the performance of SSK-SDR in the high-SNR region.

\section{Simulation Results}

To evaluate the benefits of the proposed technique, this section presents numerical results based on Monte Carlo simulations of conventional SSK without scaling (termed as SSK in the figures), SSK using the constellation randomization of [25] with $D=20$ candidate scaling vectors, termed as SSK-CR, the iterative algorithms developed in [26], and the proposed SSK using SDR optimization, namely SSK-SDR. In this section we maintain the nomenclature of [26] for coherence, i.e. the algorithm employed to solve $\mathcal{P}_{1}$ is referred to as the SSK maximum minimum distance (SSK-MMD) regime, whereas $\mathcal{P}_{2}$ is solved via the so-called SSK guaranteed Euclidean distance (SSK-GED) algorithm. Throughout this section the convergence of the above SSK-MMD and SSKGED algorithms is deemed to be achieved when the Euclidean norm of the error between the input and the output of the convex optimization problem performed every iteration satisfies $\xi \leq 10^{-3}$. The performance of the systems constructed with the aid of the non-relaxed optimization problems is not shown due to their intractable computational complexity. The channel impulse response follows $\mathbf{H} \in \mathbb{C}^{N_{r} \times N_{t}} \sim \mathcal{C N}\left(\mathbf{0}, \mathbf{I}_{N_{r}} \otimes \mathbf{I}_{N_{t}}\right)$ and is assumed to be perfectly known at the transmitter and the receiver unless otherwise stated. In the previous expression, $\otimes$ denotes the Kronecker product. Without loss of generality, for the conventional techniques and for the case of problem $\mathcal{P}_{1}^{\prime}$ of the proposed scheme we assume that the average transmit power is restricted to $P_{t} \leq 1$, unless stated otherwise. MIMO systems having four TAs are considered, albeit the benefits of the proposed technique can also be extended to the SM-aided large-scale multi-user systems of [45], [46].

First, we characterize the BER performance upon increasing the transmit SNR for a $(4 \times 2)$-element MIMO system in Fig. 3. In this case, the comparison involves the MED maximization problem $\mathcal{P}_{1}^{\prime}$ upon varying the noise power. It can be seen in Fig. 3 that while SSK-CR improves the conventional SSK transmission by the TPS operation, the proposed scheme further enhances the attainable performance by the proposed optimization. Simultaneously, it can be seen that the SSKMMD algorithm of [26] offers a slight improvement over the proposed SSK-SDR in the scenario considered. However, we note that this is achieved at the cost of a significantly higher computational complexity, since multiple convex optimization problems must be solved, before convergence is achieved. This is explicitly shown in Table II, where both the average and the standard deviation of the computational time required to obtain the transmit pre-scaling factors are portrayed for $10^{4}$ channel realizations. These results indicate that SSK-SDR is capable of offering a similar performance at a computational time up to 20 times shorter than that required by the SSK-MMD algorithm [26]. Indeed, it can be observed that the proposed SSK-SDR scheme offers a significantly smaller variation in the computational time required for solving the optimization problems

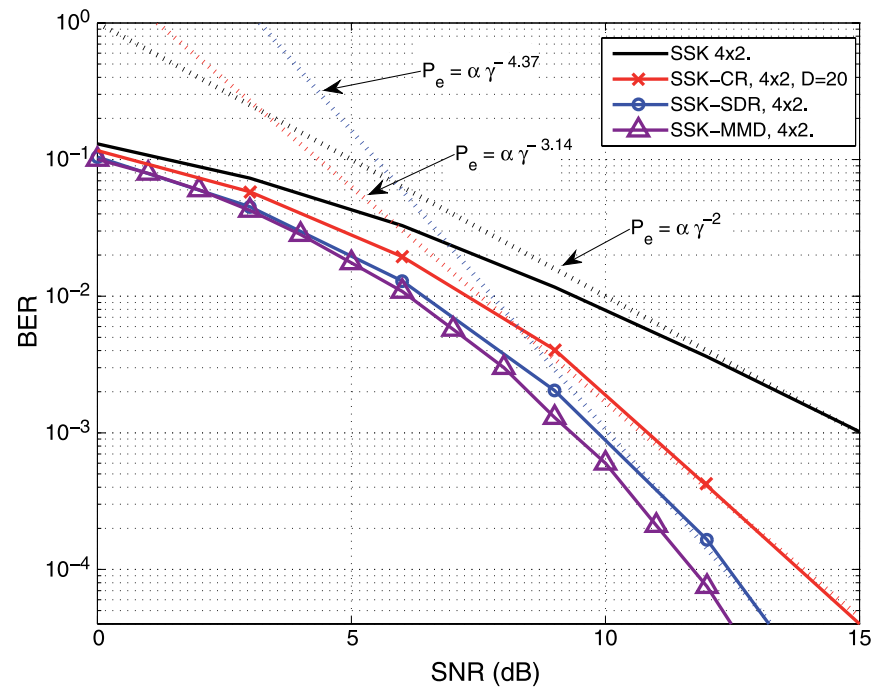

Fig. 3. BER vs. SNR for a $(4 \times 2)$ MIMO system.

TABLE II

Average Computational Time Employed to Compute THE TRANSMIT PRE-SCALING FACTORS WITH OPTIMIZATION-BASED ALGORITHMS

\begin{tabular}{|c||c|c||c|c|}
\hline$N_{t} / N_{r}$ & \multicolumn{2}{|c|}{ SSK-MMD } & \multicolumn{2}{c|}{ SSK-SDR } \\
\hline \hline & $\mu$ & $\sigma$ & $\mu$ & $\sigma$ \\
\hline $4 / 2$ & $3.85 \mathrm{sec}$. & $3.9 \mathrm{sec}$. & $0.24 \mathrm{sec}$. & $0.025 \mathrm{sec}$. \\
\hline $4 / 3$ & $4.67 \mathrm{sec}$. & $3.74 \mathrm{sec}$. & $0.23 \mathrm{sec}$. & $0.022 \mathrm{sec}$. \\
\hline
\end{tabular}

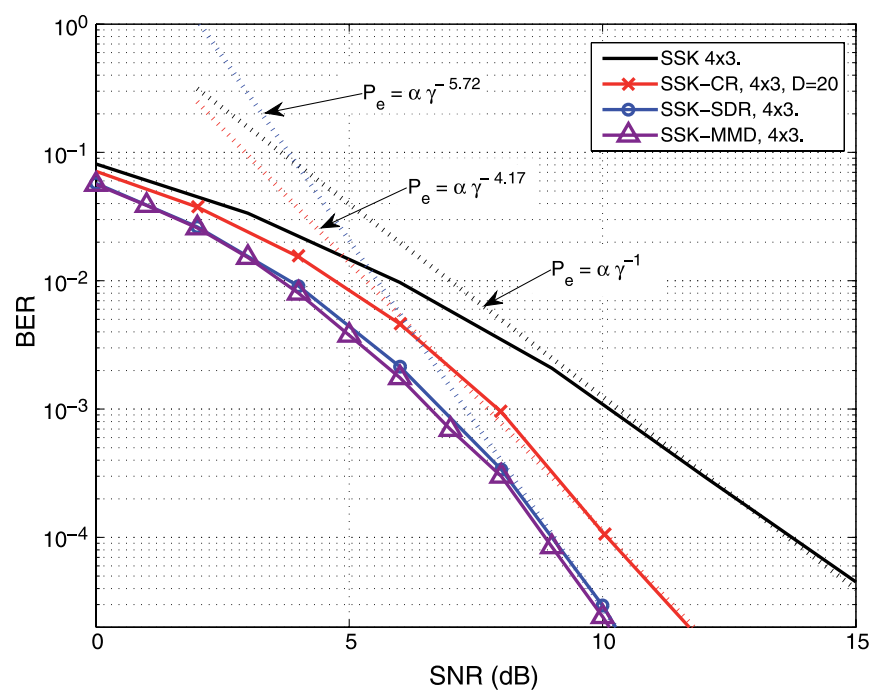

Fig. 4. BER vs. SNR for a $(4 \times 3)$ MIMO system.

under different channel conditions. Additionally, Fig. 3 portrays the high diversity order provided by SSK-SDR thanks to the enhanced MED. This is evidenced by the higher slope of the theoretical BER curve obtained by using (30), which closely matches the simulation results for sufficiently high SNR values in line with the definition of the diversity gain [47].

Similar trends can be observed in Fig. 4 , where the $(4 \times 3)$ element MIMO system is examined. In this particular case, the performance of both SSK-MMD and SSK-SDR is almost 


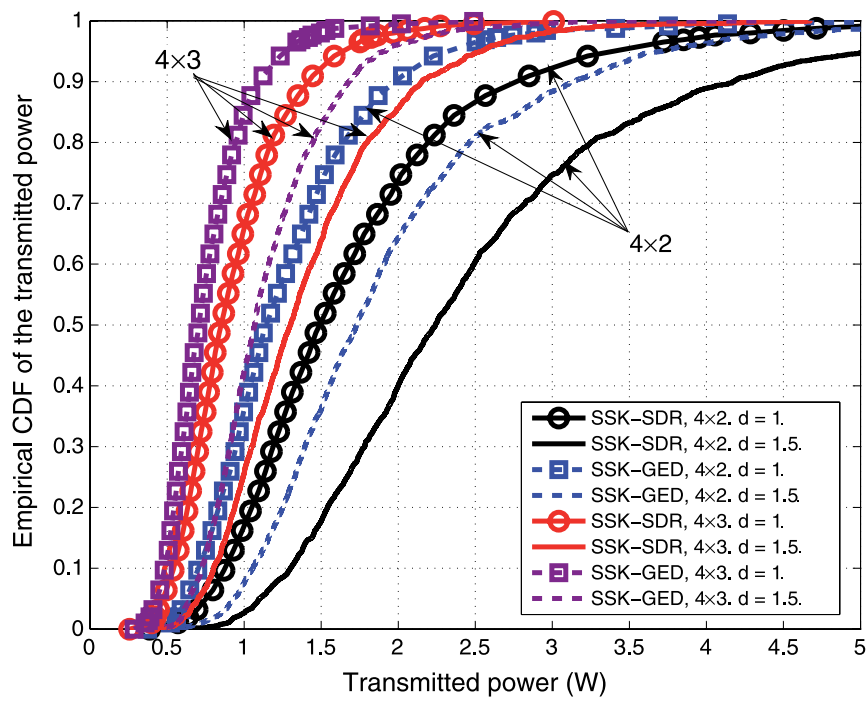

Fig. 5. Empirical CDF of transmission power for $(4 \times 2)$ and $(4 \times 3)$ MIMO systems.

indistinguishable. This entails that the proposed SSK-SDR strategy provides the best performance-complexity trade-off, since the SSK-MMD algorithm is significantly more complex as detailed in Table II.

Fig. 5 shows the cumulative distribution function (CDF) of the transmit power for problem $\mathcal{P}_{2}^{\prime}$ and SSK-GED in the above two scenarios of Figs. 3 and 4 , for different values of the MED thresholds $d$. It can be seen that the $(4 \times 3)$ element MIMO system has a smaller spread in the transmit power values necessitated for guaranteeing the required MED threshold, and this spread tends to increase upon increasing the MED thresholds $d$. It is also shown that SSK-GED generally requires less transmission power than SSK-SDR to satisfy a given MED threshold, as further analyzed in the following. It should be noted that the transmit power spread results from the fact that the MED threshold has to be satisfied under different channel conditions, which imposes a transmit power variation.

Fig. 6 illustrates the average transmit power in Watts required for increasing MED thresholds using SSK, SSK-CR, SSK-GED and the power minimization problems $\mathcal{P}_{2}^{\prime}$ and $\widetilde{\mathcal{P}}_{2}^{\prime}$ for the $(4 \times 2)$ and $(4 \times 3)$-element MIMO systems of Figs. 3 and 4 . To prevent repetition, in the following we focus our attention on the power minimization problem for clarity, although it is clear that the MED maximization problem requires a similar solution [42]. The imperfect CSI is modeled using (21) and we have $\epsilon_{j}=\epsilon=2 \tau=0.02 \forall j \in 1, \ldots N_{c}$, for the robust optimization problem $\widetilde{\mathcal{P}}_{2}^{\prime}$. The value of $\epsilon$ has been fixed similarly to [43] for illustrative purposes and without loss of generality. For the cases of SSK and SSK-CR, the scaling vector is multiplied by an appropriate factor in order to increase the separation between the estimated received constellation symbols and to guarantee that the MED constraint is satisfied with the aid of the available CSI estimate (not the true channel). Accordingly, in Fig. 6 we plot the estimated transmit power required to satisfy the MED thresholds at reception. However, we remark that the actual received symbols are different from the ones estimated at the transmitter due to the effect of the imperfect CSI. As a result,
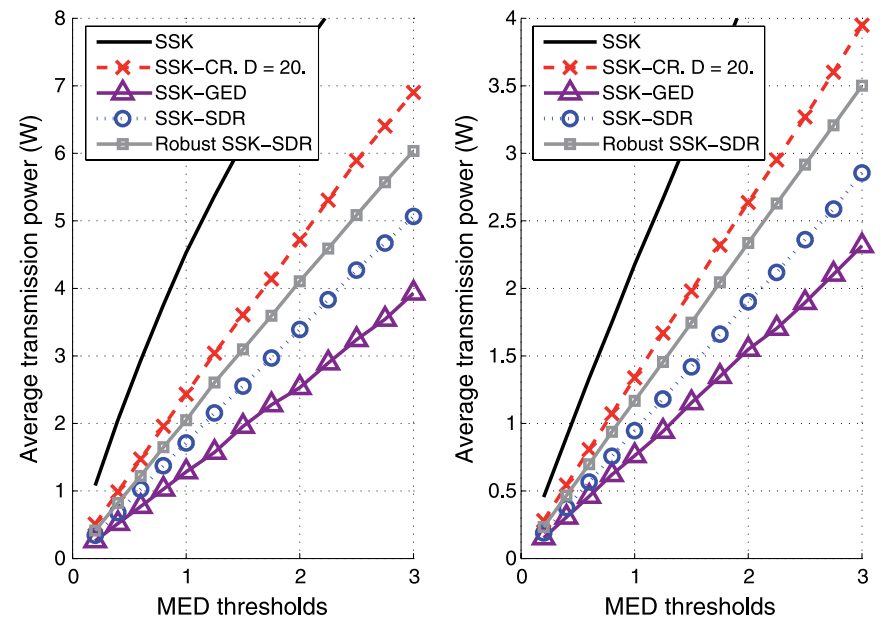

Fig. 6. Average transmission power vs MED thresholds for $(a)(4 \times 2)$ and (b) $(4 \times 3)$ MIMO systems.

achieving the true MED attained by the non-robust designs in the presence of imperfect CSI cannot be guaranteed. By contrast, the robust schemes are able to guarantee that the MED thresholds are satisfied, as detailed in Section IV. For practical reasons and without loss of generality, the transmitter has a maximum transmission power of 20 Watts for a given channel realization and we treat the solutions having higher power requirements as being infeasible. This constraint is realistic due to the technical limitations of the power amplifiers and it only takes effect for certain badly conditioned channels, since usually the transmission power is considerably lower, as shown in Fig. 6.

The results of Fig. 6 show that the proposed strategies are able to outperform SSK-CR while, simultaneously, SSK-GED is able to provide better solutions in the considered scenarios. At this point we remark that the SSK-GED approach is, however, significantly more complex than the proposed SSK-SDR. The enhancements offered by the pre-scaling techniques are a direct consequence of the solutions being closer to the optimal ones, as detailed in Section III-D. In this case, this allows us to reduce the transmission power required satisfy a given MED threshold when compared with SSK-CR. Specifically, transmit power savings as significant as 2 Watts for $d=3$ in a $(4 \times 2)$ system w.r.t. SSK-CR can be achieved, while a similar trend can also be observed in the $(4 \times 3)$ scenario. We can therefore conclude that the benefits of the pre-scaling designs become more pronounced for high MED thresholds. Moreover, similarly to [43], [48], the results of this figure show the increase in the transmit power necessary to compensate for the CSI uncertainty at the transmitter. In this particular case it can be seen that, in spite of the required transmit power increase, the insightful design offered by the robust SSKSDR design philosophy still improves upon SSK-CR, where the set of candidate scaling factors is randomly designed. By doing this, the robust SSK-SDR design guarantees that the performance thresholds specified at the receiver are satisfied, while this is not achieved by SSK, SSK-CR, SSK-GED, SSKSDR and other TPS approaches in the literature [19]-[21], [25], [26]. 

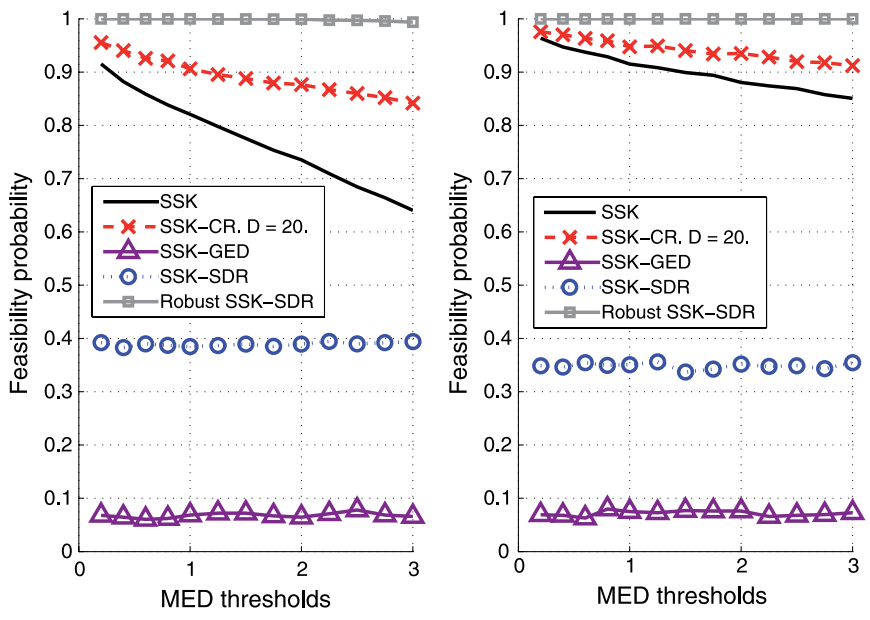

Fig. 7. Probability of feasibility vs MED thresholds for (a) $(4 \times 2)$ and (b) $(4 \times 3)$ MIMO systems.

The above observation can be explicitly seen in Fig. 7(a) and (b), where the probability of feasibility is represented for increasing values of the MED thresholds for the $(4 \times 2)$ and $(4 \times 3)$ MIMO systems, respectively. The feasibility probability is defined as $\operatorname{Prob}(\mathrm{MED} \geq d)$, provided that the average transmission power required does not exceed 20 Watts for a given channel realization. The remaining cases are considered as infeasible solutions. The same simulation parameters have been used to obtain the results of this figure as in Fig. 6. By analyzing the results of Fig. 7 it can be concluded that the proposed robust strategy offers the highest reliability, since the probability of outage is reduced w.r.t. the non-robust alternatives that do not account for the presence of CSI errors.

A more refined picture of the systems' behaviour considered in this paper under imperfect CSI conditions is shown in Fig. 8, which represents the empirical probability density function $(\mathrm{PDF})$ of the MED at the receiver in a $(4 \times 2)$ MIMO system in the presence of imperfect CSI by considering the same channel model and conditions as those used in Fig. 6. The target MED at the receiver has been set to $d=1$ and it is denoted by the red bar in the figures. Note that the red bars are only shown for illustrative purposes and do not represent the PDF over that range. The results of this figure show that the proposed robust SSK-SDR design is capable of guaranteeing the MED thresholds to be exceeded at the receiver. This is in contrast with the pre-scaling designs introduced in [19]-[21], [25], [26], where achieving the system performance required cannot be guaranteed, as depicted in Fig. 8(a), (b), (c) and (d) for the conventional SSK, SSK-CR, SSK-GED, SSK-SDR and designs, respectively. Identical conclusions can be extracted for the $(4 \times 3)$ MIMO system not shown in this paper for brevity. Accordingly, our robust technique enables the application of TPS with MED guarantees in the case of imperfect CSI, which cannot be achieved by the previous approaches. Moreover, the benefits of the proposed robust design can be clearly seen upon comparing Fig. 8(b) and (d). In particular, it can be seen that the robust technique requires less transmission power than SSK-CR, as shown in Fig. 6 and, simultaneously, the MEDs at the receiver are significantly enhanced. This improvement comes at the cost of an increased variance in the MED at the receiver w.r.t. the SSK-GED and SSK-SDR designs characterized in 8(c) and (d), which, however, have a higher outage probability due to ignoring the impact of inaccurate CSI [48].

\section{CONCLUSION}

A TPS vector design based on convex optimization has been proposed for SSK systems. A pair of distinct optimization problems has been introduced, namely MED maximization under transmit power budget constraints and transmit power minimization under a MED constraint. By invoking SDR, a closeto-optimal TPS has been designed for improving the power efficiency of SSK systems with respect to existing approaches by offering an enhanced performance for a given transmit power budget, or equivalently, a reduced transmit power for achieving a given MED. Moreover, a robust design has been developed that is capable of guaranteeing the received MED target in the presence of CSI errors at the transmitter, hence enabling the use of TPS strategies, when realistic imperfect CSI is available.

Future work can be carried out by applying the above methodology to SM, by additionally taking into account the classically modulated symbols in the structure of the constraint matrices. The employment of other robust design criteria and the combination with other strategies such as those in [17] also constitute promising subjects for our future study.

\section{APPENDIX A \\ PROOF OF THEOREM 1}

The proof of the theorem is based on exploiting the resemblance between the optimization constraints of $\widetilde{\mathcal{P}}_{1}$ and the robust beamforming designs developed in [43], [49], and on reformulating the optimization constraints with the aid of the Lagrange dual function [43], [44], [48]. Specifically, we commence by reformulating the optimization constraints in the second line of (26), which constitute the optimal solution of the optimization problem for a given value of the optimization variable $\mathbf{X}$

$$
\begin{array}{ll}
\underset{\boldsymbol{\Delta}_{j} \in \mathcal{C}_{j}}{\operatorname{minimize}} & \operatorname{Tr}\left(\left(\widetilde{\mathbf{C}}_{j}^{\prime}+\boldsymbol{\Delta}_{j}\right) \mathbf{X}\right)-d \\
\text { subject to } & \widetilde{\mathbf{C}}_{j}^{\prime}+\boldsymbol{\Delta}_{j} \succeq 0,\left\|\boldsymbol{\Delta}_{j}\right\|_{F}^{2} \leq \epsilon_{j}, j=1, \ldots, N_{c} .
\end{array}
$$

This is a convex optimization problem associated with the Lagrange dual function given by [34]

$$
\begin{array}{r}
g\left(\lambda_{j}, \mathbf{B}_{j}\right)=\inf _{\boldsymbol{\Delta}_{j}}\left\{L\left(\boldsymbol{\Delta}_{j}, \lambda_{j}, \mathbf{B}_{j}\right)\right\}=\inf _{\boldsymbol{\Delta}_{j}}\left\{\operatorname{Tr}\left(\left(\widetilde{\mathbf{C}}_{j}^{\prime}+\boldsymbol{\Delta}_{j}\right) \mathbf{X}\right)\right. \\
\left.-d+\lambda_{j}\left(\left\|\boldsymbol{\Delta}_{j}\right\|_{F}^{2}-\epsilon_{j}^{2}\right)-\operatorname{Tr}\left(\left(\widetilde{\mathbf{C}}_{j}^{\prime}+\boldsymbol{\Delta}_{j}\right) \mathbf{B}_{j}\right)\right\}
\end{array}
$$

where inf $\{\cdot\}$ denotes the infimum of a function, $L\left(\boldsymbol{\Delta}_{j}, \lambda_{j}, \mathbf{B}_{j}\right)$ is the Lagrangian, while $\lambda_{j}$ and $\mathbf{B}_{j}$ are dual variables [34]. The 


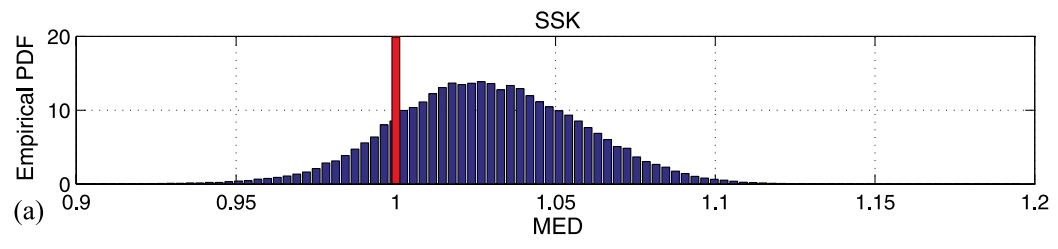

(a)

SSK-CR
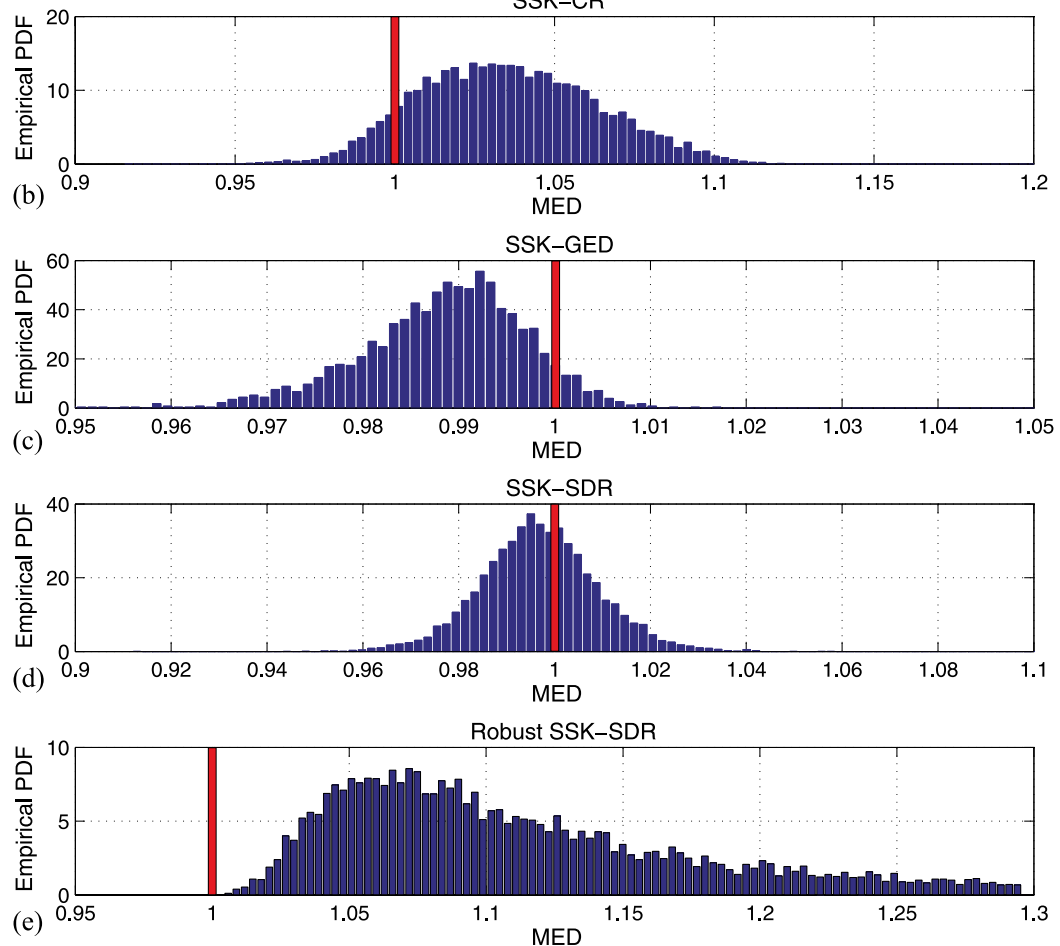

Fig. 8. Empirical PDF of the MED of a $(4 \times 2)$ MIMO system under imperfect CSI conditions with $\tau=0.01$ and $d=1$ for (a) SSK, (b) SSK-CR, (c) SSK-GED, (d) SSK-SDR, and (e) robust SSK-SDR.

infimum of the Lagrangian in (32) can be obtained by finding the point satisfying

$$
\frac{\partial L\left(\boldsymbol{\Delta}_{j}, \lambda_{j}, \mathbf{B}_{j}\right)}{\partial \boldsymbol{\Delta}_{j}}=0
$$

which is achieved for [43], [50]

$$
\boldsymbol{\Delta}_{j}=\frac{\mathbf{B}_{j}^{H}-\mathbf{X}^{H}}{2 \lambda_{j}} \text {. }
$$

Here, we remark that the particular structure of $\boldsymbol{\Delta}_{j} \in \mathcal{C}_{j}$ detailed in Section IV has to be considered, when defining the dual variable $\mathbf{B}_{j}$ [44]. This is required for preserving the sparsity that arises in the constraint matrices of the proposed optimization problem $\widetilde{\mathbf{C}}_{j}^{\prime}$. With this objective, we define the set $\mathcal{T}_{j}$ that indexes the non-zero entries of the $j$-th constraint matrix as

$$
\mathcal{T}_{j}=\left\{\left\{k_{j}, m_{j}\right\},\left\{k_{j}, k_{j}\right\},\left\{m_{j}, k_{j}\right\},\left\{m_{j}, m_{j}\right\}\right\} .
$$

In these indices represented as $\{a, b\}, a$ refers to the row and $b$ corresponds to the column of the indexed matrix. Moreover, let $\mathcal{Q}_{j}=\left\{\{r, c\} \mid r, c \in 1, \ldots, N_{t}\right\}$ denote the set containing all the indices of an $\left(N_{t} \times N_{t}\right)$-element matrix and $\mathcal{S}_{j}=\mathcal{Q}_{j}-\mathcal{T}_{j}$. Note that $\mathcal{S}_{j}$ indexes the zero-valued entries of the $j$-th con- straint matrix $\widetilde{\mathbf{C}}_{j}^{\prime}$. Following the above argument, the following relationship must hold to preserve the structure of $\boldsymbol{\Delta}_{j} \in \mathcal{C}_{j}$

$$
\left.\mathbf{B}_{j}\right|_{\mathcal{S}_{j}}=\left.\mathbf{X}\right|_{\mathcal{S}_{j}},
$$

where $\left.\mathbf{X}\right|_{\mathcal{S}_{j}}$ denotes the entries of the matrix $\mathbf{X}$ determined by $\mathcal{S}_{j}$. This guarantees that we have $\boldsymbol{\Delta}_{j} \in \mathcal{C}_{j}$, since $\left.\mathbf{B}\right|_{\mathcal{S}_{j}}-$ $\left.\mathbf{X}\right|_{\mathcal{S}_{j}}=\mathbf{0}$. For notational convenience, we define $\mathcal{B}_{j}$ as the set of matrices satisfying (36). Considering this and substituting (34) into (32), the Lagrange dual problem can be reformulated as [34]

$$
\begin{gathered}
\max _{\mathbf{B}_{j}, \lambda_{j}}-\left(\operatorname{Tr}\left(\widetilde{\mathbf{C}}_{j}^{\prime}\left(\mathbf{B}_{j}-\mathbf{X}\right)\right)+\frac{\left\|\mathbf{B}_{j}-\mathbf{X}\right\|_{F}^{2}}{4 \lambda_{j}}+\lambda_{j} \epsilon_{j}^{2}+d\right) \\
\text { subject to } \mathbf{B}_{j} \succeq 0, \mathbf{B}_{j} \in \mathcal{B}_{j}, \lambda_{j} \geq 0 .
\end{gathered}
$$

At this point, we remark that (37) is an equivalent formulation of the optimization constraints in (31) because strong duality holds, i.e. the duality gap is zero and the bound provided by the Lagrange dual problem is tight [34]. This is because the (weaker) Slater condition that entails strong duality holds, if there exists a solution satisfying $\widetilde{\mathbf{C}}_{j}^{\prime}+\boldsymbol{\Delta}_{j} \succeq 0$ and $\left\|\boldsymbol{\Delta}_{j}\right\|_{F}<$ $\epsilon_{j}$. Since $\widetilde{\mathbf{C}}_{j}^{\prime}$ is positive semidefinite by definition, the above 
condition is always satisfied and therefore there is no duality gap [34]. This entails that $\widetilde{\mathcal{P}}_{1}$ can be rewritten as

$$
\begin{array}{cl} 
& \widetilde{\mathcal{P}}_{1}: \underset{\mathbf{X}, \mathbf{B}_{j}}{\operatorname{maximize}} d \\
\text { s.t. } \max _{\mathbf{B}_{j} \in \mathcal{B}_{j}}-\left(\operatorname{Tr}\left(\widetilde{\mathbf{C}}_{j}^{\prime}\left(\mathbf{B}_{j}-\mathbf{X}\right)\right)+\epsilon_{j}\left\|\mathbf{B}_{j}-\mathbf{X}\right\|_{F}+d\right) \geq 0 \\
\\
\quad \mathbf{B}_{j} \succeq 0, j=1, \ldots, N_{c}, \\
\quad \mathbf{X} \succeq 0, \operatorname{Tr}(\mathbf{X})<=\left(P_{t} N_{t}\right), \operatorname{rank}(\mathbf{X})=1,
\end{array}
$$

where the dual problem that determines the constraints in the second line of (38) has been already maximized w.r.t. $\lambda_{j}$. Finally, Theorem 1 is obtained by noting that the constraints determined by the maximum function are immediately satisfied for any feasible $\mathbf{B}_{j} \in \mathcal{B}_{j}$ and by dropping the rank constraint, which completes the proof.

\section{REFERENCES}

[1] R. Mesleh, H. Haas, S. Sinanovic, C. W. Ahn, and S. Yun, "Spatial modulation," IEEE Trans. Veh. Technol., vol. 57, no. 4, pp. 2228-2241, Jul. 2008.

[2] M. Di Renzo, H. Haas, A. Ghrayeb, S. Sugiura, and L. Hanzo, "Spatial modulation for generalized MIMO: Challenges, opportunities, and implementation," Proc. IEEE, vol. 102, no. 1, pp. 56-103, Jan. 2014.

[3] P. Yang et al., "Single-carrier spatial modulation: A promising design for large-scale broadband antenna systems," IEEE Commun. Surveys Tuts. to be published.

[4] M. Di Renzo and H. Haas, "Bit error probability of space modulation over Nakagami-m fading: Asymptotic analysis," IEEE Commun. Lett., vol. 15 , no. 10 , pp. $1026-1028$, Oct. 2011.

[5] A. Younis, S. Sinanovic, M. Di Renzo, R. Mesleh, and H. Haas, "Generalised sphere decoding for spatial modulation," IEEE Trans. Commun., vol. 61, no. 7, pp. 2805-2815, Jul. 2013.

[6] A. Garcia-Rodriguez and C. Masouros, "Low-complexity compressive sensing detection for spatial modulation in large-scale multiple access channels," IEEE Trans. Commun., vol. 63, no. 7, pp. 2565-2579, Jul. 2015.

[7] C. Masouros and L. Hanzo, "Dual layered MIMO transmission for increased bandwidth efficiency," IEEE Trans. Veh. Technol., to be published. [Online]. Available: http://ieeexplore.ieee.org/xpl/articleDetails. jsp?arnumber $=7128734$

[8] C. Masouros and L. Hanzo, "Constructive interference as an information carrier by dual layered MIMO transmission," IEEE Trans. Veh. Technol., to be published.

[9] M. Di Renzo and H. Haas, "On transmit diversity for spatial modulation MIMO: Impact of spatial constellation diagram and shaping filters at the transmitter," IEEE Trans. Veh. Technol., vol. 62, no. 6, pp. 2507-2531, Jul. 2013.

[10] P. Yang et al., "Star-QAM signaling constellations for spatial modulation," IEEE Trans. Veh. Technol., vol. 63, no. 8, pp. 3741-3749, Oct. 2014.

[11] S. Sugiura, C. Xu, S. X. Ng, and L. Hanzo, "Reduced-complexity coherent versus non-coherent QAM-aided space-time shift keying," IEEE Trans. Commun., vol. 59, no. 11, pp. 3090-3101, Nov. 2011.

[12] K. Ntontin, M. Renzo, A. Perez-Neira, and C. Verikoukis, "Adaptive generalized space shift keying," EURASIP J. Wireless Commun. Netw., vol. 2013, no. 43, pp. 1-5, 2013.

[13] S. Sugiura and L. Hanzo, "On the joint optimization of dispersion matrices and constellations for near-capacity irregular precoded space-time shif keying," IEEE Trans. Wireless Commun., vol. 12, no. 1, pp. 380-387, Jan. 2013.

[14] M. Maleki, H. Bahrami, S. Beygi, M. Kafashan, and N. Tran, "Space modulation with CSI: Constellation design and performance evaluation," IEEE Trans. Veh. Technol., vol. 62, no. 4, pp. 1623-1634, May 2013.

[15] L.-L. Yang, "Transmitter preprocessing aided spatial modulation for multiple-input multiple-output systems," in Proc. IEEE VTC Spring, May 2011, pp. 1-5.

[16] A. Stavridis, S. Sinanovic, M. Di Renzo, and H. Haas, "Transmit precoding for receive spatial modulation using imperfect channel knowledge," in Proc. IEEE VTC Spring, May 2012, pp. 1-5.
[17] R. Y. Chang, S.-J. Lin, and W.-H. Chung, "Energy efficient transmission over space shift keying modulated MIMO channels," IEEE Trans. Commun., vol. 60, no. 10, pp. 2950-2959, Oct. 2012.

[18] R. Y. Chang, S.-J. Lin, and W.-H. Chung, "New space shift keying modulation with Hamming code-aided constellation design," IEEE Wireless Commun. Lett., vol. 1, no. 1, pp. 2-5, Feb. 2012.

[19] X. Guan, Y. Cai, and W. Yang, "On the mutual information and precoding for spatial modulation with finite alphabet," IEEE Wireless Commun Lett., vol. 2, no. 4, pp. 383-386, Aug. 2013.

[20] J. Luna-Rivera, D. U. Campos-Delgado, and M. Gonzalez-Perez, "Constellation design for spatial modulation," Procedia Technol., vol. 7, pp. 71-78, 2013.

[21] C. Masouros, "Improving the diversity of spatial modulation in MISO channels by phase alignment," IEEE Commun. Lett., vol. 18, no. 5, pp. 729-732, May 2014.

[22] M. Di Renzo and H. Haas, "Improving the performance of Space Shift Keying (SSK) modulation via opportunistic power allocation," IEEE Commun. Lett., vol. 14, no. 6, pp. 500-502, Jun. 2010.

[23] C.-H. Wu, W.-H. Chung, and H.-W. Liang, "Improved generalized spaceshift keying via power allocation," IEEE Commun. Lett., vol. 18, no. 7, pp. 1143-1146, Jul. 2014.

[24] T. Handte, A. Muller, and J. Speidel, "BER analysis and optimization of generalized spatial modulation in correlated fading channels," in Proc. IEEE VTC Fall, Sep. 2009, pp. 1-5.

[25] C. Masouros and L. Hanzo, "Constellation-randomization achieves transmit diversity for single-RF spatial modulation," IEEE Trans. Veh. Technol., to be published. [Online]. Available: http://greeninterference. weebly.com/uploads/2/4/6/5/24652439/masouro-s_sm-constrand5.pdf

[26] M.-C. Lee, W.-H. Chung, and T.-S. Lee, "Generalized precoder design formulation and iterative algorithm for spatial modulation in MIMO systems with CSIT," IEEE Trans. Commun., vol. 63, no. 4, pp. 1230-1244, Apr. 2015.

[27] Z.-Q. Luo, W.-K. Ma, A.-C. So, Y. Ye, and S. Zhang, "Semidefinite relaxation of quadratic optimization problems," IEEE Signal Process. Mag. vol. 27, no. 3, pp. 20-34, May 2010

[28] J. Jeganathan, A. Ghrayeb, and L. Szczecinski, "Spatial modulation: optimal detection and performance analysis," IEEE Commun. Lett., vol. 12, no. 8, pp. 545-547, Aug. 2008.

[29] J. Jeganathan, A. Ghrayeb, L. Szczecinski, and A. Ceron, "Space shift keying modulation for MIMO channels," IEEE Trans. Wireless Commun., vol. 8, no. 7, pp. 3692-3703, Jul. 2009.

[30] C. Masouros and G. Zheng, "Exploiting known interference as green signal power for downlink beamforming optimization," IEEE Trans. Signal Process., vol. 63, no. 14, pp. 3628-3640, Jul. 2015.

[31] S. Wagner, R. Couillet, M. Debbah, and D. Slock, "Large system analysis of linear precoding in correlated MISO broadcast channels under limited feedback," IEEE Trans. Inf. Theory, vol. 58, no. 7, pp. 4509-4537, Jul. 2012.

[32] N. Sidiropoulos, T. Davidson, and Z.-Q. Luo, "Transmit beamforming for physical-layer multicasting," IEEE Trans. Signal Process., vol. 54, no. 6, pp. 2239-2251, Jun. 2006.

[33] Z. Luo, N. D. Sidiropoulos, P. Tseng, and S. Zhang, "Approximation bounds for quadratic optimization with homogeneous quadratic constraints," SIAM J. Optim., vol. 18, no. 1, pp. 1-28, 2007.

[34] S. Boyd and L. Vandenberghe, Convex Optimization. New York, NY, USA: Cambridge Univ. Press, 2004.

[35] P. Biswas and Y. Ye, "Semidefinite programming for ad hoc wireless sensor network localization," in Proc. IEEE 3rd Int. Symp. IPSN, Apr. 2004, pp. 46-54.

[36] M. Grant, S. Boyd, and Y. Ye, CVX: Matlab Software for Disciplined Convex Programming, 2008. [Online]. Available: http://cvxr.com/cvx/

[37] E. Karipidis, N. Sidiropoulos, and Z.-Q. Luo, "Quality of service and max-min fair transmit beamforming to multiple cochannel multicast groups," IEEE Trans. Signal Process., vol. 56, no. 3, pp. 1268-1279, Mar. 2008.

[38] Z. Xu, M. Hong, and Z.-Q. Luo, "Semidefinite approximation for mixed binary quadratically constrained quadratic programs," SIAM J. Optim., vol. 24, no. 3, pp. 1265-1293, 2014.

[39] A. Barvinok, "Problems of distance geometry and convex properties of quadratic maps," Discr. Comput. Geom., vol. 13, no. 1, pp. 189-202, 1995.

[40] A. Shapiro, "Rank-reducibility of a symmetric matrix and sampling theory of minimum trace factor analysis," Psychometrika, vol. 47, no. 2, pp. 187-199, Jun. 1982.

[41] G. Pataki, "On the rank of extreme matrices in semidefinite programs and the multiplicity of optimal eigenvalues," Math. Oper. Res., vol. 23, no. 2, pp. 339-358, May 1998. 
[42] A. Gershman, N. Sidiropoulos, S. ShahbazPanahi, M. Bengtsson, and B. Ottersten, "Convex optimization-based beamforming," IEEE Signal Process. Mag., vol. 27, no. 3, pp. 62-75, May 2010.

[43] I. Wajid, Y. Eldar, and A. Gershman, "Robust downlink beamforming using covariance channel state information," in Proc. IEEE ICASSP, Apr. 2009, pp. 2285-2288.

[44] B. Chalise and L. Vandendorpe, "Optimization of MIMO relays for multipoint-to-multipoint communications: Nonrobust and robust designs," IEEE Trans. Signal Process., vol. 58, no. 12, pp. 6355-6368, Dec. 2010.

[45] X. Wu, M. Di Renzo, and H. Haas, "A novel multiple access scheme based on spatial modulation MIMO," in Proc. IEEE Int. Workshop CAMAD, Dec. 2014, pp. 285-289.

[46] X. Wu, M. Di Renzo, and H. Haas, "Direct transmit antenna selection for transmit optimized spatial modulation," in Proc. IEEE VTC Fall, Sep. 2013, pp. 1-5.

[47] Y. Jiang, M. Varanasi, and J. Li, "Performance analysis of ZF and MMSE equalizers for MIMO systems: An in-depth study of the high SNR regime," IEEE Trans. Inf. Theory, vol. 57, no. 4, pp. 2008-2026, Apr. 2011.

[48] I. Wajid, M. Pesavento, Y. Eldar, and D. Ciochina, "Robust downlink beamforming with partial channel state information for conventional and cognitive radio networks," IEEE Trans. Signal Process., vol. 61, no. 14, pp. 3656-3670, Jul. 2013

[49] M. Bengtsson and B. Ottersten, "Optimal and suboptimal transmit beamforming," in Handbook of Antennas in Wireless Communications. L. C. Godara, Ed. Boca Raton, FL, USA: CRC Press, 2001.

[50] K. B. Petersen and M. S. Pedersen, "The matrix cookbook," Tech. Univ. Denmark, Lyngby, Denmark, vol. 450, pp. 7-15, 2008.

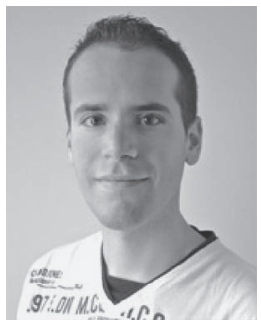

Adrian Garcia-Rodriguez (S'13) received the M.S. degree in telecommunications engineering from the Universidad de Las Palmas de Gran Canaria, Spain, in 2012. Currently, he is working toward the Ph.D. degree in the Department of Electrical \& Electronic Engineering, University College London, U.K. His research interests lie the field of signal processing and wireless communications with emphasis on energy-efficient and multi-antenna communications.

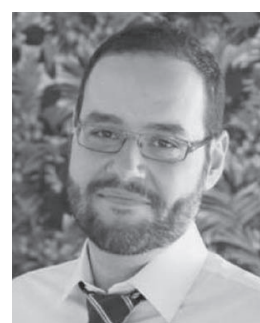

London, U.K.

He holds a Royal Academy of Engineering Research Fellowship 2011-2016 and is the Principal Investigator of the EPSRC project EP/M014150/1 on large scale antenna systems. His research interests lie in the field of wireless communications and signal processing with particular focus on green communications, large scale antenna systems, cognitive radio, interference mitigation techniques for MIMO and multicarrier communications. He is an Associate Editor for IEEE COMMUNICATIONS LETTERS.

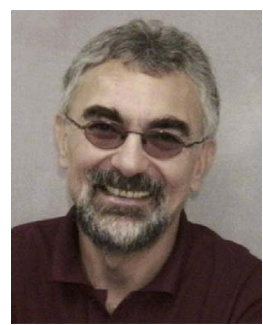

Lajos Hanzo (M'91-SM'92-F'04) received the bachelor's degree in electronics in 1976 and the doctorate degree in 1983. In 2009, he was awarded the honorary doctorate Doctor Honoris Causa by the Technical University of Budapest. During his 38-year career in telecommunications, he has held various research and academic posts in Hungary, Germany, and the U.K. Since 1986, he has been with the School of Electronics and Computer Science, University of Southampton, U.K., where he holds the Chair in telecommunications. He has successfully supervised about 100 Ph.D. students, co-authored 20 John Wiley/ IEEE Press books on mobile radio communications totalling in excess of 10,000 pages, published over 1400 research entries at IEEE Xplore, acted both as TPC and General Chair of IEEE conferences, presented keynote lectures and has been awarded a number of distinctions. Currently, he is directing a 100 -strong academic research team, working on a range of research projects in the field of wireless multimedia communications sponsored by industry, the Engineering and Physical Sciences Research Council (EPSRC) U.K., the European Research Council's Advanced Fellow Grant and the Royal Society's Wolfson Research Merit Award. He is an enthusiastic supporter of industrial and academic liaison and he offers a range of industrial courses. He is also a Governor of the IEEE Vehicular Technology Society. During 2008-2012, he was the Editor-in-Chief of the IEEE Press and a Chaired Professor also at Tsinghua University, Beijing. His research is funded by the European Research Council's Senior Research Fellow Grant. He has over 20000 citations. For further information on research in progress and associated publications, please refer to http://www-mobile.ecs.soton.ac.uk. 